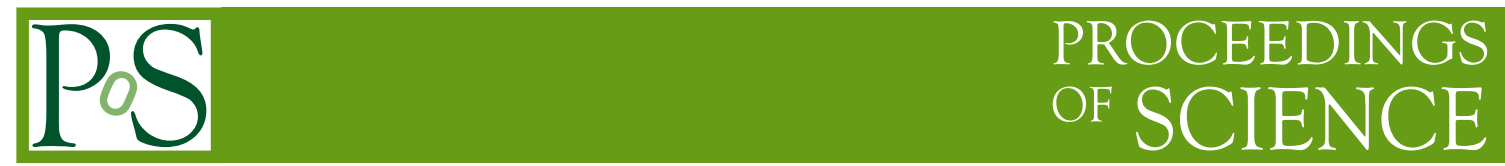

\title{
Noise, sensitivity, dynamic range and image fidelity
}

\section{R. Soria-Ruiz*}

Observatorio Astronómico Nacional, c/ Alfonso XII 3, 28014 Madrid, Spain

E-mail: r.soriadoan.es

We review the importance and impact of the noise in the data, the dynamic range and the image fidelity in the synthesis imaging process. A summary of the main sources of errors is given and how to minimize the effects of these errors in the calibration of the data.

2nd MCCT-SKADS Training School. Radio Astronomy: fundamentals and the new instruments August 26-September 4, 2008

Sigüenza, Spain

*Speaker. 


\section{Noise and sensitivity}

Noise can be defined as a random signal that mixes with the transmitted or received signal from the stellar source that we are observing. Since this undesired signal limits the quality of any communication system, it is particularly important to minimize the noise level and its effect on the observational data. Several sources may add noise to the stellar source radiation. These include external artificial noise (for example from the engines of the telescopes), atmospheric noise, noise from the ground and cosmic background radiations. In addition, the receivers and its components also introduce noise to the data. The latter is the main contribution to the total noise, and it is usually measured as the receiver temperature. In most of the radio-bands, the thermal noise, which is due to the fluctuations of the electrons in conductors, is the main contribution to the total electronic noise. Thus, in radio-astronomy the output noise power of the receiver is given by the Johnson-Nyquist equation:

$$
P_{N}=k T_{s y s} \Delta v
$$

where $T_{s y s}$ is the sum of the receiver temperature and the antenna temperature $\left(T_{A}\right)$, which measures the power absorbed by the antenna. In an ideal, loss-free radio telescope, $T_{A}$ is the brightness temperature $\left(T_{B}\right)$ if the intensity of the received radiation is constant within the main lobe, or $T_{B} \mathrm{x}\left(\Omega_{S} / \Omega_{A}\right)$, which takes into account the difference between the angular dimension of the source and that of the main lobe.

Another important issue related to the noise is the signal-to-noise-ratio (SNR). This quantity determines the noise limit of a source signal. The SNR can be defined as:

$$
S N R=\frac{\text { signal }}{\text { noise per measurement }} \sqrt{\text { number of independent measurements }}
$$

Since the source signal is given by $T_{A}$, and the noise per measurement is proportional to the power noise, thus proportonial to $T_{s y s}$, equation (1.2) can be written as

$$
S N R_{\text {lim }}=\frac{T_{A}}{T_{\text {sys }}} \sqrt{\text { number of independent measurements }}
$$

Or, inverting and dividing by the signal, the noise limit can be defined as

$$
\Delta T_{\text {lim }}=\frac{T_{\text {sys }}}{\sqrt{\text { number of independent measurements }}}
$$

On the other hand, how many independent measurements are made by a single baseline for total time $t$ over a bandwidth $\Delta v$ ? This magnitude is given by the Nyquist theorem:

$$
\text { Number of independent measurements }=2 \Delta v t
$$

Defining the antenna temperature (Rayleigh-Jeans regime) as

$$
T_{A}=\frac{\eta_{a} A}{2 k} S_{v}
$$


where $\eta_{a}$ and $A$ are the efficiency and aperture of the antenna, the noise level (Jy) on a single baseline will be

$$
\Delta S=\frac{\sqrt{2} k T_{s y s}}{\eta_{a} A \sqrt{\Delta v t}}
$$

If the system efficiency (which accounts for the electronics and digital losses) is included, renaming some terms we have:

$$
\Delta S=\frac{1}{\eta_{s}} \frac{S E F D}{\sqrt{2 \Delta v t}} \quad S E F D=\frac{T_{s y s}}{K}
$$

where SEFD (System Equivalent Flux Density) measures the overall antenna performance and K $(\mathrm{K} / \mathrm{Jy})$ is the gain of the antenna. With all this information, we can calculate the sensitivity of a synthesis image, which corresponds to the combined sensitivity of all the possible interferometer combinations. This noise limit will determine the weakest feature that can be detected in the absence of other imaging limitations. The determination of this magnitude will depend on the recording and subsequent correlation of the data. If the observational setup includes only single polarization data, the image sensitivity is the standard deviation of the mean of the number of samples $(L)$, each with a standard deviation $\Delta S$.

$$
\Delta I_{m}=\frac{\Delta S}{\sqrt{L}}
$$

Since $L=\frac{1}{2} N(N-1) \frac{t_{i n t}}{t}$ (for an homogeneous array) where $\mathrm{N}$ is the number of antennas of the array, the sensitivity of the single polarization image will be

$$
\Delta I_{m}=\frac{1}{\eta_{s}} \frac{S E F D}{\sqrt{N(N-1) \Delta v t_{\text {int }}}}
$$

If dual polarization data are recorded, the four Stokes parameters (I, Q, U and V) can be determined and so do the I, Q, U and V images. Since these images obey Gaussian statistics, in this case, the sensitivity of the image will be

$$
\Delta I=\frac{\Delta I_{m}}{\sqrt{2}}=\frac{1}{\eta_{s}} \frac{S E F D}{\sqrt{2 N(N-1) \Delta v t_{\text {int }}}}
$$

\section{Image fidelity and dynamic range}

Both, image fidelity and dynamic range are measure of the quality of an image but the link between the two quantities is hard to make. While the image fidelity is the difference between any produced image and the correct image, the dynamic range can be defined as the ratio between the peak brightness on the image and the rms in a region believed to be void of emission, that is, the dynamic range is an estimation of the image fidelity. On the other hand, the dynamic range is much easier to measure then the image fidelity because firstly, the on-source errors are typically much higher than the off-source rms, and secondly, because the true source image is unknown. 


\section{$3 C 84$ WSRT $21 \mathrm{~cm}$}
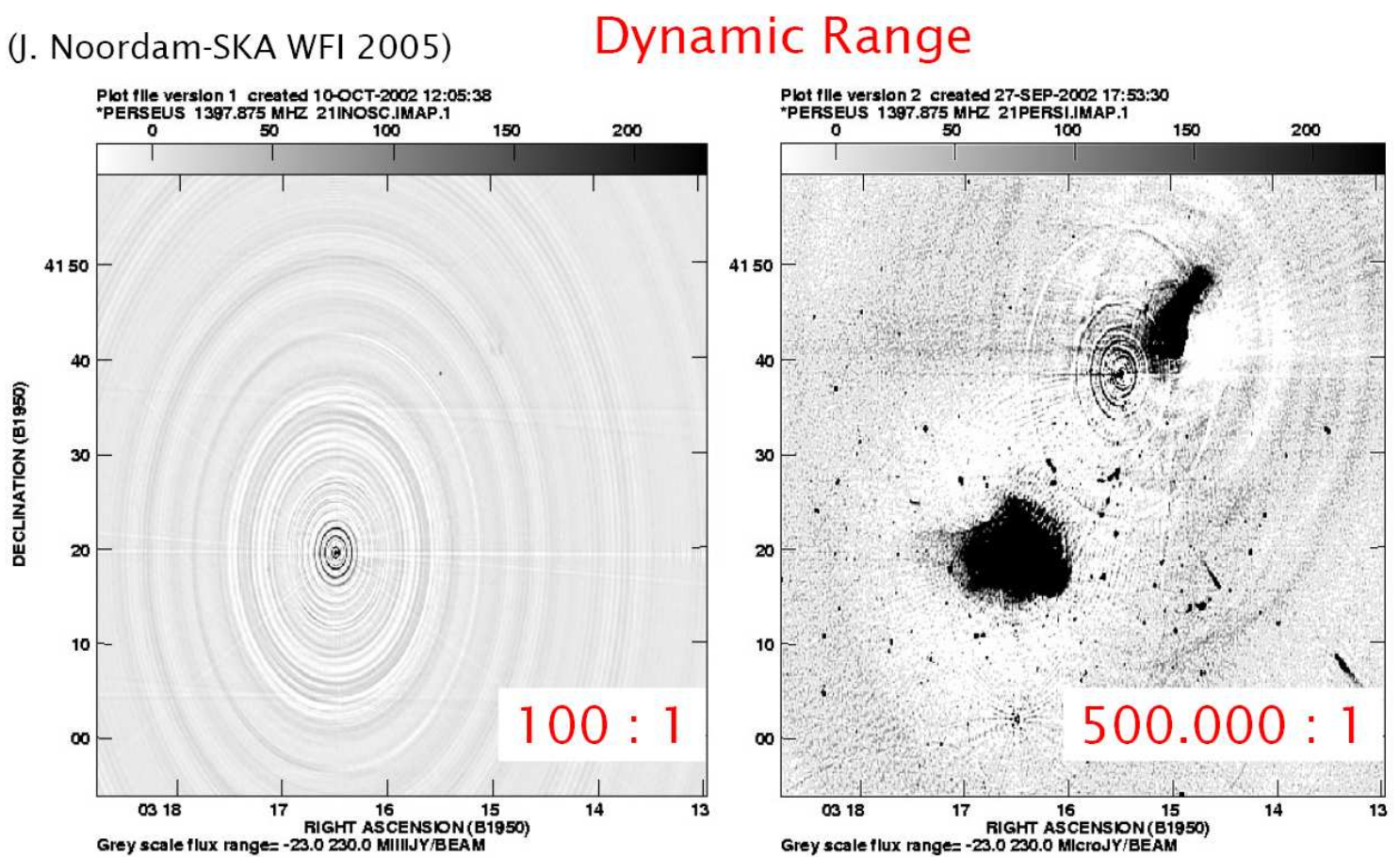

Although it is important to note that some spectacular images have low dynamic range, in general, a high dynamic range is desirable (an example is given in the following figure, where the dynamic range is 500000). By performing high quality imaging of strong sources, we are able to study the flux evolution and motions of the different components, or for example, detect weak features. In addition, the imaging of weak sources near strong sources allow us to deal with strongest sources in deep surveys and with confusing sources near specific targets. Obviously a high fidelity imaging is also desirable because that means getting the correct answer. An incorrect image can be caused by many different problems, such as errors in the data, errors in the imaging process and errors in the methodology. Since it is not possible to get the perfectly correct image, the problem resides in minimizing the sources of error. Of course, the list of potential sources of errors which limit the accuracy of imaging is very long. The important ones, as mentioned in Section 1 are the thermal noise, atmospheric phase errors (minimized by self-calibrating the data if high SNR and a good model is provided), UV coverage errors due to gaps in the coverage, loss due to phase winding (decorrelation due to coherent integration longer than the coherence time), phase or amplitude bandpass errors, etc. Many of these errors can be minimized by a deep and careful calibration and, if possible, by subsequent self-calibration.

\section{References}

[1] Proceedings from the 1993 NRAO Summer School, (Socorro), Edited by J. A. Zensus, P. J. Diamond, and P. J. Napier ASP Conference Series, Vol. 82, 1995

[2] J. Noordam, Square Kilometre Array Workshop, Wide Field Imaging, 2005, ASTRON (The Netherlands) 